\title{
Investigation of proteome of Baikal endemic amphipod Eulimnogammarus cyaneus (Dybowsky, 1874) using LC-MS
}

\author{
D. Bedulina ${ }^{1 *}$, S. Kalkhof ${ }^{2}$, A.Gurkov $^{1}$, P. Drozdova ${ }^{1}$, M. Timofeyev ${ }^{1}$, T. Luckenbach ${ }^{3}$ \\ ${ }^{1}$ Irkutsk State University, Irkutsk, Russia \\ ${ }^{2}$ The University of Applied Sciences, Coburg, Germany \\ ${ }^{3}$ Helmholtz Centre for Environmental Research, Leipzig, Germany \\ *e-mail:daria.bedulina@gmail.com
}

Key words: proteogenomics, amphipoda, Baikal, eco-proteomics, LC-MS proteomics

Motivation and Aim: Lake Baikal is unique ancient ecosystem, currently threatened by the anthropogenic impact and climate change [1]. Thus, developing of the monitoring bioassays for this lake if of the great importance. Aim of the current study was to investigate proteome of a potential model biotest species for Lake Baikal - amphipod Eulimnogammarus cyaneus (Dybowsky, 1874), using a proteogenomic approach.

Methods and Algorithms: We used 1D-PAGE of total protein lysate of E. cyaneus, followed by gel fragmentation, in-gel trypsinolysis and nano-HPLC/nano-ESI-MS/MS using LTQ Orbitrap XL ${ }^{\text {TM }}$ (Thermo Fisher Scientific). Transcriptome assembly of the respective species was used to generate a specific database for protein identification, which was carried out with MaxQuant software [2].

Results: MS-spectra were assigned to 1028 protein groups. Among them the first five major groups were belonging to the following families (identified by InterProScan Ids): tubulins, ubiquitin, heat shock protein $70 \mathrm{kDa}$, actin and hemocyanin. Used approach allowed us to validate approximately $9 \%$ of the transcriptome. Label free quantification using MaxQuant revealed 16 differently expressed ( $p<0.05$, Bonferroni correction) protein groups in males and females of the studied species.

Conclusion: Thus, for Baikal endemic amphipod E. cyaneus experimentally-validated proteomic database was established and a sexual dimorphism of proteomes was investigated. This database will allow to carry out a broad spectrum of eco-physiological and eco-toxicological researches with amphipods and to reveal new specific biomarkers of anthropogenic impact to the unique ecosystem of Lake Baikal.

Acknowledgements: Supported by the RFBR (14-01-00885, 14-01-31134), and Grant of the President of the Russian Federation (MK-6804.2018.4).

\section{References}

1. Timoshkin O.A., Samsonov, D.P., Yamamuro, M., Moore, M.V., Belykh, O.I., Malnik, V.V., ... Fedorova G.A. (2016) Rapid ecological change in the coastal zone of Lake Baikal (East Siberia): Is the site of the world's greatest freshwater biodiversity in danger? J. Great Lakes Research. 42(3):487-497.

2. Cox J., Mann M. (2008) MaxQuant enables high peptide identification rates, individualized ppb-range mass accuracies and proteome-wide protein quantification. Nature Biotechnol. 26(12):1367. 